
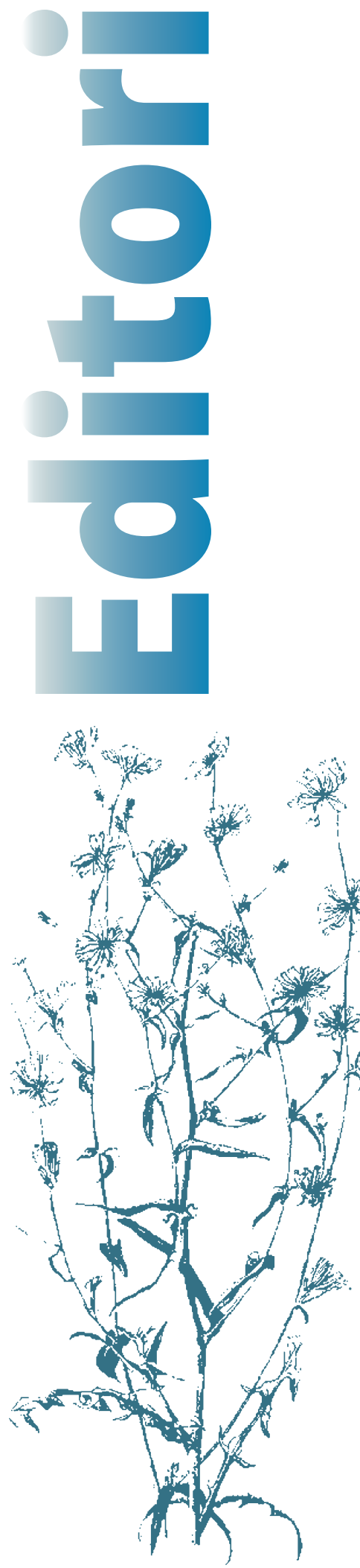

\title{
Trop de critiques tuent le bon sens
}

Les médicaments à base de Ginkgo biloba $(\mathrm{Gb})$ ont fait l'objet d'une réévaluation de leurs indications en ophtalmologie, en ORL, en cardiologie et en neurologie. "Leur efficacité a été jugée insuffisante dans leurs indications respectives ", explique l'Afssaps. Et de préciser : "Ils présentent des effets indésirables majoritairement non graves, de type digestifs et cutanés ". Bien sûr : "Après examen des données d'efficacité et de sécurité d'emploi, la Commission d'AMM a conclu que le rapport bénéfice/risque de ces médicaments était défavorable ». L’Agence va prochainement adresser une lettre aux professionnels de santé pour leur faire part de cet avis.

Pourtant, les spécialistes, ORL, en ophtalmologie et neurologie, prescrivent le Gb ! Et cela, bien qu'ils n'aient pas de formation en phytothérapie. Ce constat apparaît paradoxal, et il serait surprenant que l'activité de ces médicaments se limite aux seuls effets traditionnels...

Il est bien connu que le Gb est peu vasodilatateur et qu'il a une action sur les structures nerveuses (oreille, cerveau, nerf périphérique) sur le long court et un effet sur le vasculaire (l'artère comme contenta, le liquide sanguin comme contenu).

La Commission E n'est pas tendre en général ni pour les effets élagués ni pour la toxicologie et déclare :

\section{" Excerpted from the Complete Commission E Monographs:} Therapeutic Guide to Herbal Medicine

\section{Ginkgo biloba ( $G$ b) Leaf Extract Ginkgo folium (published July 19, 1994)}

Composition of Drug (A dry extract from the dried leaf of Gb L. manufactured using acetone/water and subsequent purification steps without addition of concentrates or isolated ingredients. The drug/extract ratio is $35-67: 1$, on average 50:1).

The extract is characterized by: 22-27 percent flavone glycosides, determined as quercetin and kaempferol, including isorhamnetin (via HPLC) and calculated as flavones with a molar mass of $\mathrm{MMr}=756.7$ (quercetin glycosides) and $\mathrm{Mr}=740.7$ (kaempferol glycosides); 5-7 percent terpene lactones, of which approximately 2.8-3.4 percent consists of ginkgolides A, B and C, as well as approximately 2.6-3.2 percent bilobalide; below $5 \mathrm{ppm}$ ginkgolic acids. The given ranges include manufacturing and analytical variances.

Pharmacological properties (Pharmacokinetics, Toxicology)

The following pharmacological effects have been established experimentally: - improvement of hypoxic tolerance, particularly in the cerebral tissue; 
Les inconvénients du Gb sont connus depuis une vingtaine d'années. Les effets secondaires les plus répandus sont : plaintes gastriques, nausée, dyspepsie, réactions allergiques cutanées. Comme le Gb agit comme anti-PAF, l'administration de fluidifiants sanguins (aspirine, antiplaquettaires, AVK), nécessite des précautions d'emploi.

Bien sûr, les évaluations évoluent en fonction d'une meilleure connaissance des résultats avec un produit ainsi qu'avec les relevés de pharmacovigilance. Il y aurait lieu de distinguer les différents produits dérivés du Gb, notamment l'extrait sélectif titré qui 
relève d'une analyse à part; ce qui ne semble pas fait. De même, il est important de dissocier les aspects économiques de remboursement de l'évaluation scientifique qui ne sont pas directement liés même si l'évaluation défavorable peut avoir a posteriori des conséquences. Il s'agit bien du travail de commissions différentes (transparence et $A M M)$. Une telle note de l'Afssaps sans distinction entre les différents produits dérivés du Gb préjuge, en général, l'arrêt total du remboursement des produits qui le contiennent et non une note explicative aux médecins susceptibles de prescrire.

Le système est actuellement fondé sur les preuves par la science, c'est-à-dire les métaanalyses et l'analyse des résultats des essais cliniques via les outils statistiques... Si le système est bien fondé et adapté pour l'étude d'une substance isolée sur une cible, ce n'est plus du tout le cas pour une préparation complexe qui agit sur plusieurs cibles et/ou de manière systémique.

Les flavonoïdes, et plus généralement les polyphénols, ont des propriétés non spécifiques telles quantioxydantes ou d'interactions avec les enzymes qui se traduisent par des effets globaux difficiles à mettre en évidence par les méthodes d'évaluation classiques. Le plus complexe est l'activité génomique, c'est-à-dire les interactions avec l'expression des gènes : nutrigénomique et/ou pharmacogénomique... C’est la raison pour laquelle il importe de travailler à démontrer les limites du système actuel en particulier les biais et incertitudes liées à la modélisation excessive. La complexité du biologique ne peut complètement être évaluée par les essais cliniques ni les méta-analyses...

Curieusement, il y a dans notre Union européenne des divergences de vue non seulement dans les finances mais aussi dans les opinions de scientifiques. Avec la "crise", betaucoup d’organismes publics perdent du crédit. Alors que les centres européens considèrent que le $\mathrm{Gb}$ a sa place dans des maladies neurodégénératives, notre instance officielle le déclare inefficace. À qui accorder du crédit ? Il serait temps que le vrai débat ait lieu entre médecins, professionnels de santé (pharmaciens), scientifiques,

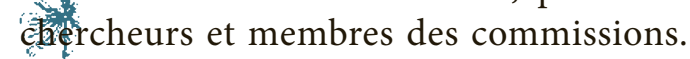

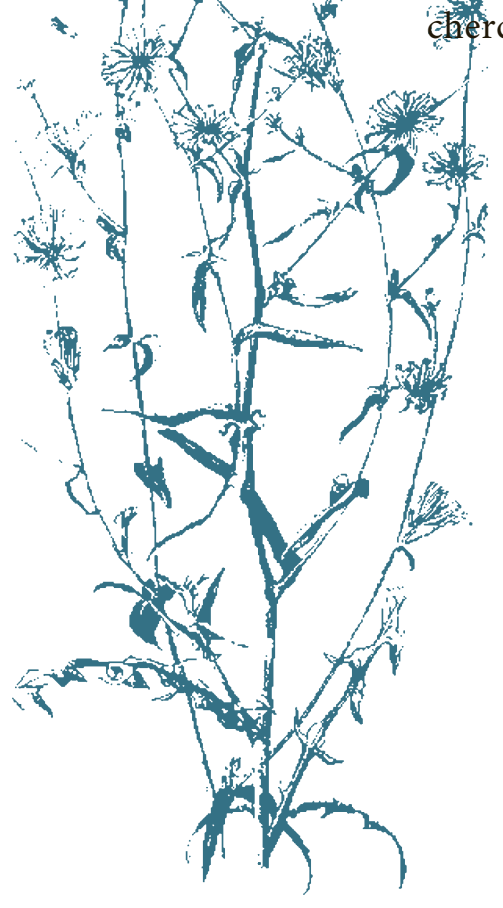

Dr Paul Goetz

Rédacteur en chef

Lö̈c Bureau

Responsable formation IFAS, Pr de nutraceutique UFR sciences pharmaceutiques, université Rennes-I 